\title{
Amplified Spontaneous Emission and Optical Gain in Organic Single Crystal Quinquethiophene
}

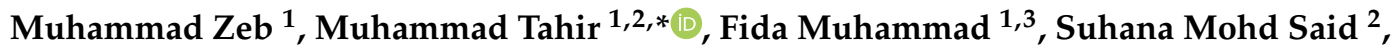 \\ Mohd Faizul Mohd Sabri ${ }^{4}$, Mahidur R. Sarker ${ }^{4}$, Sawal Hamid Md Ali ${ }^{5}$ and Fazal Wahab ${ }^{6}$ (D) \\ 1 Department of Physics, Faculty of Physical and Numerical Sciences, Abdul Wali Khan University Mardan, \\ 23200 Khyber Pakhtunkhwa, Pakistan; mzebphy@gmail.com (M.Z.); fida_physics@hotmail.com (F.M.) \\ 2 Department of Electrical Engineering, Faculty of Engineering, University of Malaya, 50603 Kuala Lumpur, \\ Malaysia; smsaid@um.edu.my \\ 3 Electronics \& Nanoscale Engineering, School of Engineering, University of Glasgow, Glasgow G12 8QQ, UK \\ 4 Department of Mechanical Engineering, Faculty of Engineering, University of Malaya, 50603 Kuala Lumpur, \\ Malaysia; faizul@um.edu.my (M.F.M.S.); mahidur@um.edu.my (M.R.S.) \\ 5 Department of Electric, Electronics and System Engineering, Faculty of Engineering and Built Environment, \\ Universiti Kebangsaan Malaysia, Bangi 43600, Malaysia; sawal@ukm.edu.my \\ 6 Department of Physics, Karakoram International University, Gilgit 15100, Pakistan; fazalwhb@gmail.com \\ * Correspondence: tahir@awkum.edu.pk or mtahirphys@um.edu.my; Tel.: +60-3-79677022
}

Received: 9 September 2019; Accepted: 2 October 2019; Published: 21 November 2019

check for updates

\begin{abstract}
In this paper, we report optical characteristics of an organic single crystal

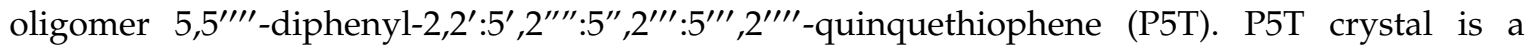
thiophene/phenylene co-oligomer that possesses better charge mobility as well as photoluminescence quantum efficiency (PLQE) as compared to other organic materials. Stimulated emission in P5T is investigated via amplified spontaneous emission (ASE) measurements within broad pump energies ranging from 35.26 to $163.34 \mu \mathrm{J} / \mathrm{cm}^{2}$. An Nd-YAG femtosecond-tunable pulsed laser is used as a pump energy source for the ASE measurements of P5T crystals at an excitation wavelength of $445 \mathrm{~nm}$. The ASE spectra exhibit optical amplification in P5T crystals at a $625 \mathrm{~nm}$ peak wavelength with a lower threshold energy density $\left(\mathrm{E}_{\mathrm{th}}\right) \approx 52.64 \mu \mathrm{J} / \mathrm{cm}^{2}$. P5T also demonstrates higher optical gain with a value of $72 \mathrm{~cm}^{-1}$, that is calculated by using the variable stripe-length method. The value of PLQE is measured to be $68.24 \%$ for P5T. This study proposes potential applications of P5T single crystals in organic solid state lasers, photodetectors, and optical amplifiers.
\end{abstract}

Keywords: amplified spontaneous emission (ASE); optical gain; PLQE; P5T single crystal; thiophene/phenylene co-oligomer

\section{Introduction}

Organic semiconductors have received significant attention from researchers and scientists due to their potential applications in various electronic and optoelectronic devices such as organic light emitting diodes (OLEDs) [1-3], organic light emitting field effect transistors (OLEFETs) [4,5], organic photovoltaics (OPV) [6,7], and organic field effect transistors (OFETs) [8,9]. A few of the interesting characteristics of organic semiconductors are their low cost, low temperature processability, light weight, mechanical flexibility, and solubility in various organic solvents [10-12]. Most of the organic semiconductors are either amorphous or polycrystalline, due to which they have usually lower charge carrier mobility as compared to inorganic semiconductors. Therefore, the charge mobility in organic materials needs to be improved [13] for its potential applications in high performance devices such as OLEDs, photodetectors, and solid state lasers. One of the characteristics that can enhance charge mobility is the purity and crystal nature of the semiconductor materials [14]. However, a particular class 
of organic copolymers and co-oligomers demonstrates molecular crystal structure with a higher charge mobility as compared to other organic materials [15]. Such crystalline organic materials are the best alternatives to the most expensive and highest temperature-processed inorganic semiconductors [16].

Amongst the organic copolymers, thiophene/phenylene copolymers are made of thiophene and benzene rings in-line as backbone, which possesses higher charge mobility, is single-crystal in nature, and has high potential for optoelectronic devices $[15,17]$. Because of these extraordinary properties, thiophene/phenylene copolymers demonstrate spectrally narrow emissions, when optically pumped, and also are the best candidates for OLEFETs [18]. Hence, it is the periodic order of molecules in these thiophene/phenylene copolymer crystals that is responsible for the improved physical properties and makes them perfect for promising device applications $[15,19]$. They offer remarkable performance in metal-free lasing mode which reduces the quenching effect as reported by De Villers [20-22].

Optical gain has been studied in many organic semiconductors such as methyl-substituted ladder-type poly(p-phenylene) (MeLPPP), polyfluorine derivatives, and poly(p-phenylene-vinylenes) (PPVs) [23,24]. The materials, which demonstrate amplified spontaneous emission (ASE), can produce a laser if the proper resonator is applied and enough thickness of film is available to sustain waveguide modes. The ASE occurs when a film/material is optically excited by a short light pulse beyond its threshold energy and, consequently, a remarkable contraction of the emission spectrum is observed. ASE is a direct technique to study the photophysics of active materials and also identify controlling factors for optical gain $[25,26]$. Under the quest for exploring more suitable organic semiconductors for solid state lasers, OFETs, and OLEDs, a plethora of organic materials has been researched by optimizing the thickness of the active film, optical pumping-wavelength, charge mobility, and morphology etc. [27]. However, thin films based on single crystals with highly regular arrangements of molecules reveal promising optoelectronic and photonic properties. Furthermore, single crystal semiconductors offer outstanding photonic properties, for instance, self-wave guided photoemission, and high internal as well as photoluminescence quantum efficiency (PLQE) [28].

Among the thiophene/phenylene co-oligomer single crystals, $5,5^{\prime \prime \prime \prime}$-diphenyl-2, $2^{\prime}: 5^{\prime}, 2^{\prime \prime}: 5^{\prime \prime}, 2^{\prime \prime \prime}: 5^{\prime \prime \prime}, 2^{\prime \prime \prime \prime}$-quinquethiophene (P5T) is an oligomer single crystal that possesses interesting photonic, electronic, and optoelectronic properties which make it one of the best candidates for OLEDs, OFETs, and solid state lasers [18]. P5T has essentially enhanced charge transport properties due to its strong intermolecular forces produced by sulphur as compared to nonsulphur molecules. Usually, a longer thiophene chain shows higher luminescence performance as well as a broad spectrum adjustability [29]. Apart from this, better performance has been observed in the organic semiconductor single crystals as compared to polycrystalline and/or amorphous materials. For example, in OFET configurations, higher charge carrier mobilities up to $40 \mathrm{~cm}^{2} /$ V.s were observed [30] while better results in polarized emissions and laser oscillations have also been reported [31].

In this work, we study P5T crystal deposited on quartz glass (P5T/glass) as a wave-guide medium and for optical amplification through ASE measurements and optical gain characteristics. The ASE threshold energy and photoluminescence quantum efficiency (PLQE) measurements of P5T are also performed. The value of optical gain is calculated by the stripe-length method.

\section{Experimental Works}

The single crystals of P5T were obtained on quartz glass according to the scheme reported elsewhere [15,32]. The molecular weight of P5T was $564.83 \mathrm{~g} / \mathrm{mol}$ whereas the molecular structure and its crystals' actual images are shown in Figure 1a,b, respectively. The images were obtained by using a NIKON ELIPSE LV100POL microscope (Nikon Instruments Europe BV, Amsterdam,, Netherlands) with an attached mercury $(\mathrm{Hg})$ lamp. For ASE measurements, a 3-dimensional stage was used where the P5T/quartzglass sample was fixed on sample holder. For optical excitation, a $445 \mathrm{~nm}$ wavelength was shone on the P5T crystal from a tuneable pulsed laser source (EKSPLA NT342 B-SH) of Neodymium Yttrium Aluminum Garnate (Nd:YAG) having a repetition rate of $10 \mathrm{~Hz}$ with a pulse duration of $5 \mathrm{~ns}$. 
A neutral density filter was used to control the incident pulse energy which was measured with a thermoelectric detector (Scientech, Colorado, USA). By using some slits and cylindrical lens, the laser beam was targeted perpendicular to the P5T sample. The beam profile such as the spot size or strip size was measured to be $0.0431 \mathrm{~cm}^{2}$ as shown in Figure 1c.

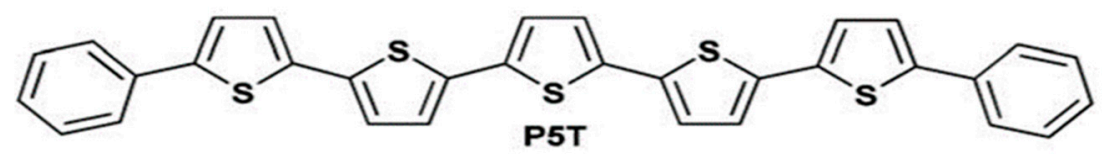

(a)


$\underline{400 \mu \mathrm{m}}$

(c)

Figure 1. (a) Molecular structure of P5T (b) optical micrographs of P5T crystals at different optical polarizations (c) femtosecond laser excitation beam profile/spot size.

To control the laser beam profile, a telescope and proper slits were used while CCD (Win Cam D, Gentec) was used for measuring the beam profile/spot size. The PL spectrum was recorded from the edge of the P5T/glass sample, directed to the slit of a monochromator, and collected by an intensified CCD camera (Toshiba TCD1304AP Linear CCD array, Toshiba, Tokyo, Japan) and Ocean Fiber Optic Spectrometer USB4000 (Ocean Optics Inc., Ostfildern, Germany,). Also, ASE intensity was measured as a function of excitation stripe-length for optical gain measurements. The PLQE of the sample was measured using an integrating-sphere connected to a Spex FluoroMax 3 fluorometer (Horiba, Kyoto, Japan). All the measurements were carried out at ambient conditions and $300 \mathrm{~K}$. The schematic diagram of the ASE setup is shown in Figure 2. 


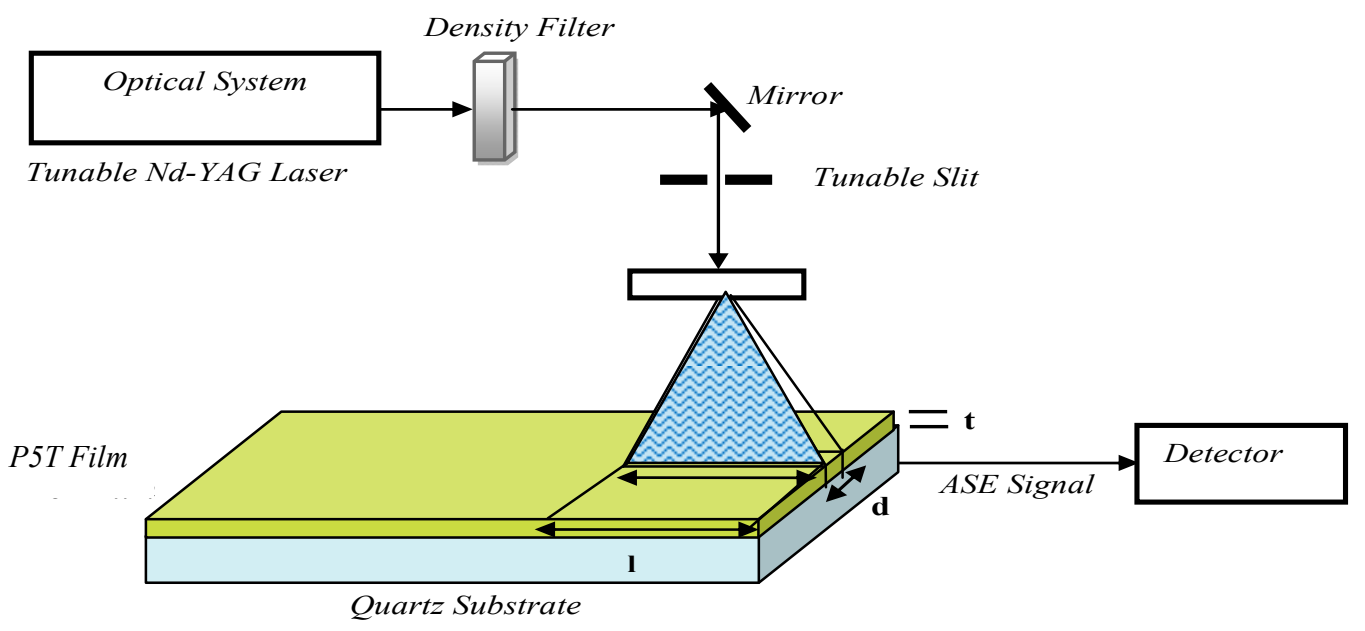

Figure 2. Schematic of the diagram amplified spontaneous (ASE) setup.

\section{Results}

The PL spectra measurements of the P5T crystal were carried out to study the stimulated emission. A pumping wavelength of $445 \mathrm{~nm}$ was been used to excite the P5T crystal. The emission spectra were measured at different energies (from $35.26 \mu \mathrm{J} / \mathrm{cm}^{2}$ to $163.34 \mu \mathrm{J} / \mathrm{cm}^{2}$ ) where the spectral lines narrowing effect could be observed as the energy was increased from lower to higher as shown in Figure 3. This narrowing of spectral lines designates the presence of ASE and optical gain in the P5T crystal. The spontaneous emission amplification occurred at peak intensity at $625 \mathrm{~nm}$ emission wavelength. Besides from the line narrowing effect, the output intensity was also increased. Each spectrum in Figure 3 is labeled with the corresponding energy.

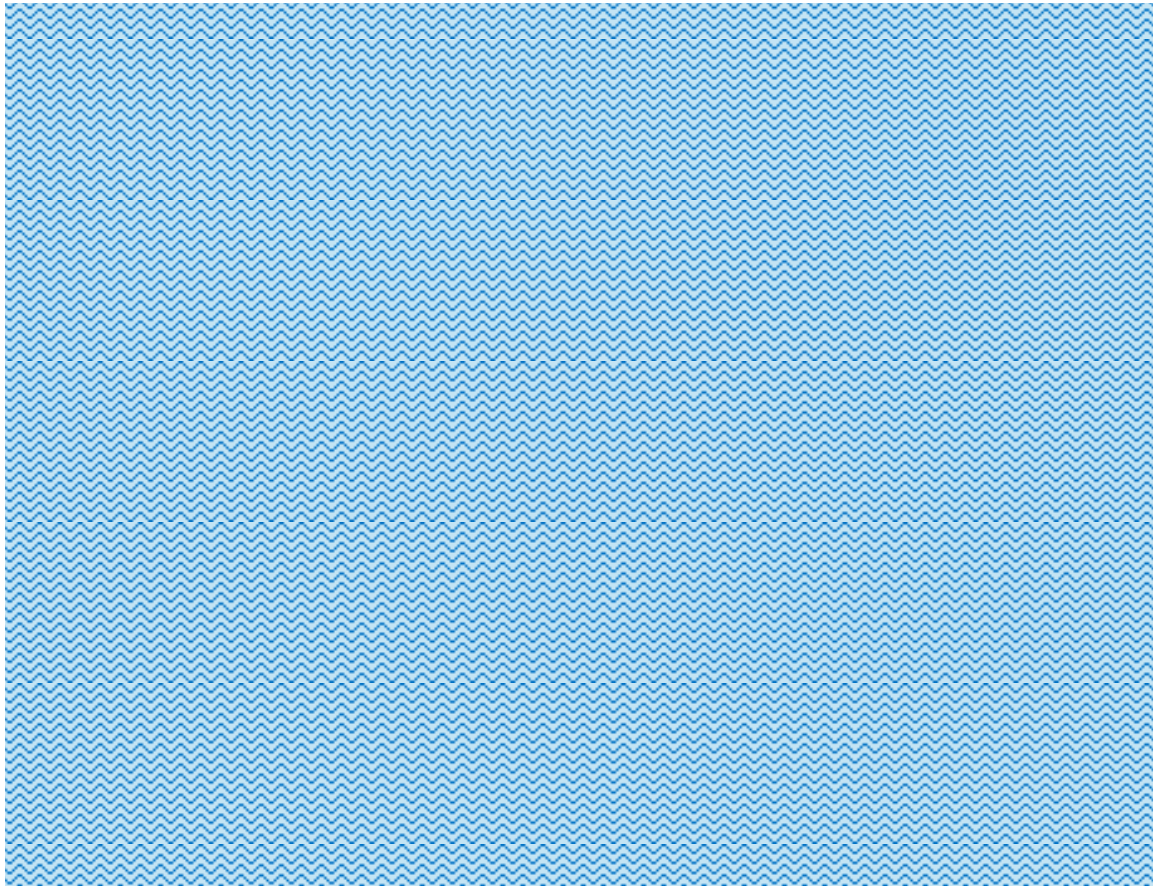

Figure 3. Emission spectra of the P5T single crystal pumped at an excitation wavelength of $445 \mathrm{~nm}$ with different pulsed energies.

The spectral lines' narrowing and ASE phenomenon is also evident from the full width half maximum (FWHM) curves plotted against the pump energy density, as shown in Figure 4. The FWHM is getting decreased as the excitation energy is increasing. The trend of decrease of the FWHM is 
weaker at low excitation energies between $35.26-52 \mu \mathrm{J} / \mathrm{cm}^{2}$, however, as the pumping energy was further increased beyond $52 \mu \mathrm{J} / \mathrm{cm}^{2}$ there was an approximately linear and dominant shift in the FWHM towards a narrowing effect. This linear and rapid narrowing is due to the supply of pumping energy above the threshold energy $\left(E_{t h}\right)$ required for optical amplification and stimulated emission in the P5T crystal.

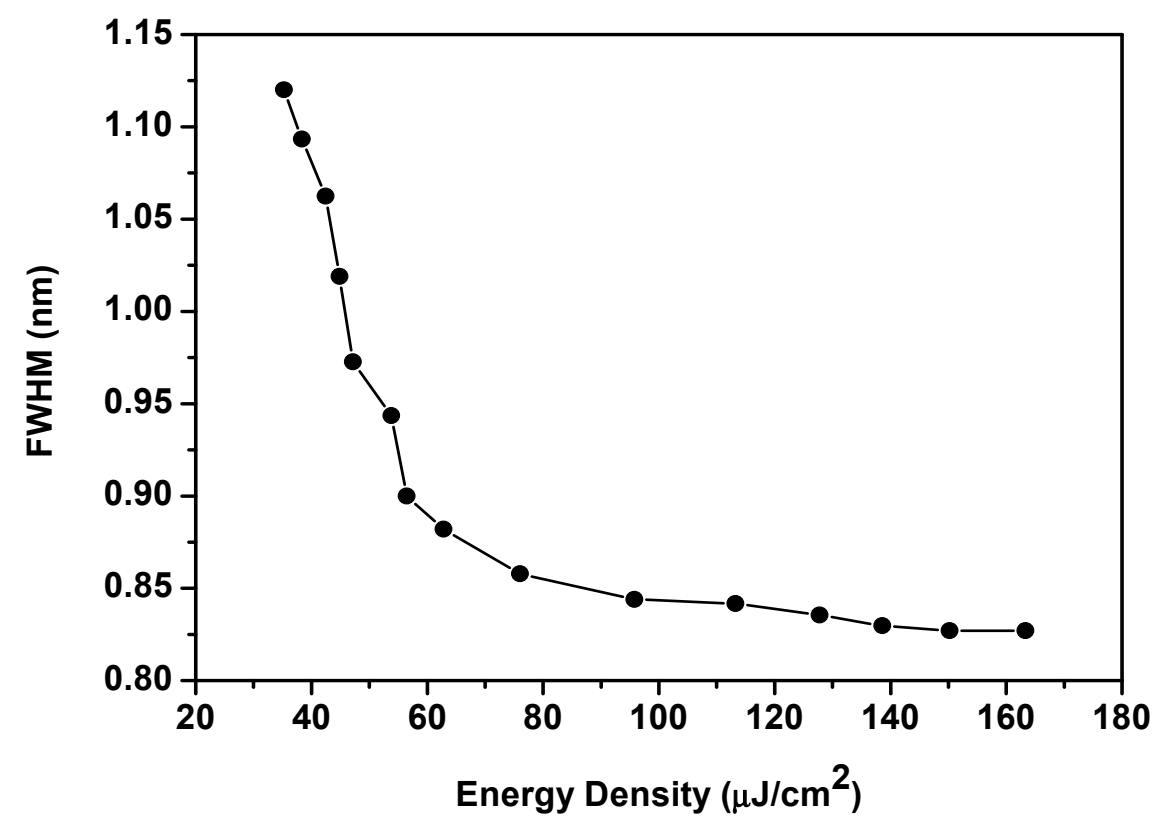

Figure 4. Full width half maximum (FWHM)-energy density relation for the P5T crystal.

Figure 5 demonstrates the output intensity at the peak emission wavelength $(625 \mathrm{~nm})$ as a function of the pumping/excitation energy. Figure 5 is plotted by using data from the Figure 3 where at different excitation energies there are corresponding curves which all peak at $625 \mathrm{~nm}$. Figure 5 shows that when the pumping/excitation energy is increased the output intensity is also increased, however, at a particular energy, the output intensity shows a notable increase in the slope. This particular pumping intensity is known as the "threshold energy $\left(E_{t h}\right)$ " [33]. The value of $E_{\text {th }}$ can also be obtained from the curves showing the emission line width of FWHM versus pumping intensity [34]. The ASE threshold energy " $\mathrm{E}_{\mathrm{th}}$ " is extracted from Figure 5 and is found to be $52.64 \mu \mathrm{J} / \mathrm{cm}^{2}$ for the P5T crystal, which is smaller (better) than many organic semiconductors [28]. 


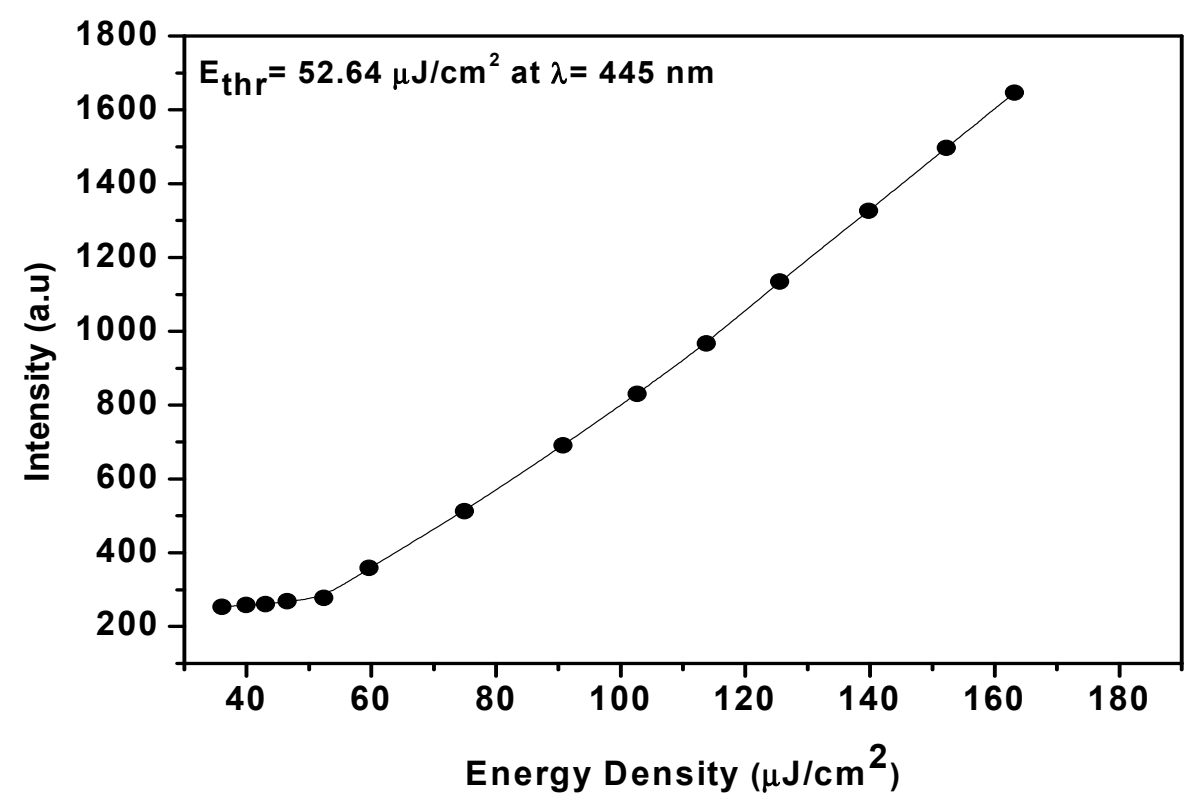

Figure 5. The ASE threshold energy characteristics of P5T at a $445 \mathrm{~nm}$ excitation wavelength.

The variable stripe-length (VSL) method was used to measure the optical gain in P5T. In this method, the pumping energy above the $E_{\text {th }}$ was provided to the sample whereas length of the sample exposed to the pumping wavelength $(445 \mathrm{~nm})$ was varied slowly and the output intensity was collected at the edge of the sample. Hence, the output intensity was characterized as a function of the stripe-length. If the PL was measured as a function of the incident intensity, then line narrowing occurred at the peak gain wavelength and ASE was confirmed. If ASE was achieved at short stripe-lengths, the spectrum would have been broad, and as the excitation length increased the spectrum would have got narrow. The output intensity depends exponentially on stripe-length which is given by equation (1) [35]. By the VSL method, stripe-length vs. output intensity curves of P5T is shown in Figure 6,

$$
\mathrm{I}(\lambda)=\frac{\mathrm{A}(\lambda) \mathrm{I}_{\mathrm{p}}}{\mathrm{g}(\lambda)}\left(\mathrm{e}^{\mathrm{g}(\lambda) l}-1\right)
$$

where $A(\lambda)$ is a constant related with the cross-section for spontaneous emission, $I_{p}$ is the incident intensity, $g(\lambda)$ is the gain coefficient, and $l$ is the length of the targeted stripe. The net $g(\lambda)$ was calculated by fitting the curves shown in Figure 6 to the expected dependence for ASE in the small-signal regime. When the excited stripe does not affect the emission width then super-fluorescence and bi-excitonic emissions are the mechanisms that are responsible for line narrowing, and the output intensity linearly increases with the excited stripe. The value of $g(\lambda)$ measured for the P5T crystal was $72 \mathrm{~cm}^{-1}$ which is much better than those measured for other organic materials, such as $22 \mathrm{~cm}^{-1}$ for poly-(9,9-dioctylfluorene-alt-benzothiadiazole) (F8BT), $13 \mathrm{~cm}^{-1}$ for oligo-(p-phenylenevinylene), and $16 \mathrm{~cm}^{-1}$ for $\mathrm{N}, \mathrm{N}^{\prime}$-bis(3-methylphenyl)-N,N'-diphenylbenzidine (TPD) [33,36]. The large value of $g(\lambda)$ suggests the potential of P5T crystals for potential applications in optical amplifiers, solid state lasers, OLEFETs, and OLEDs. 


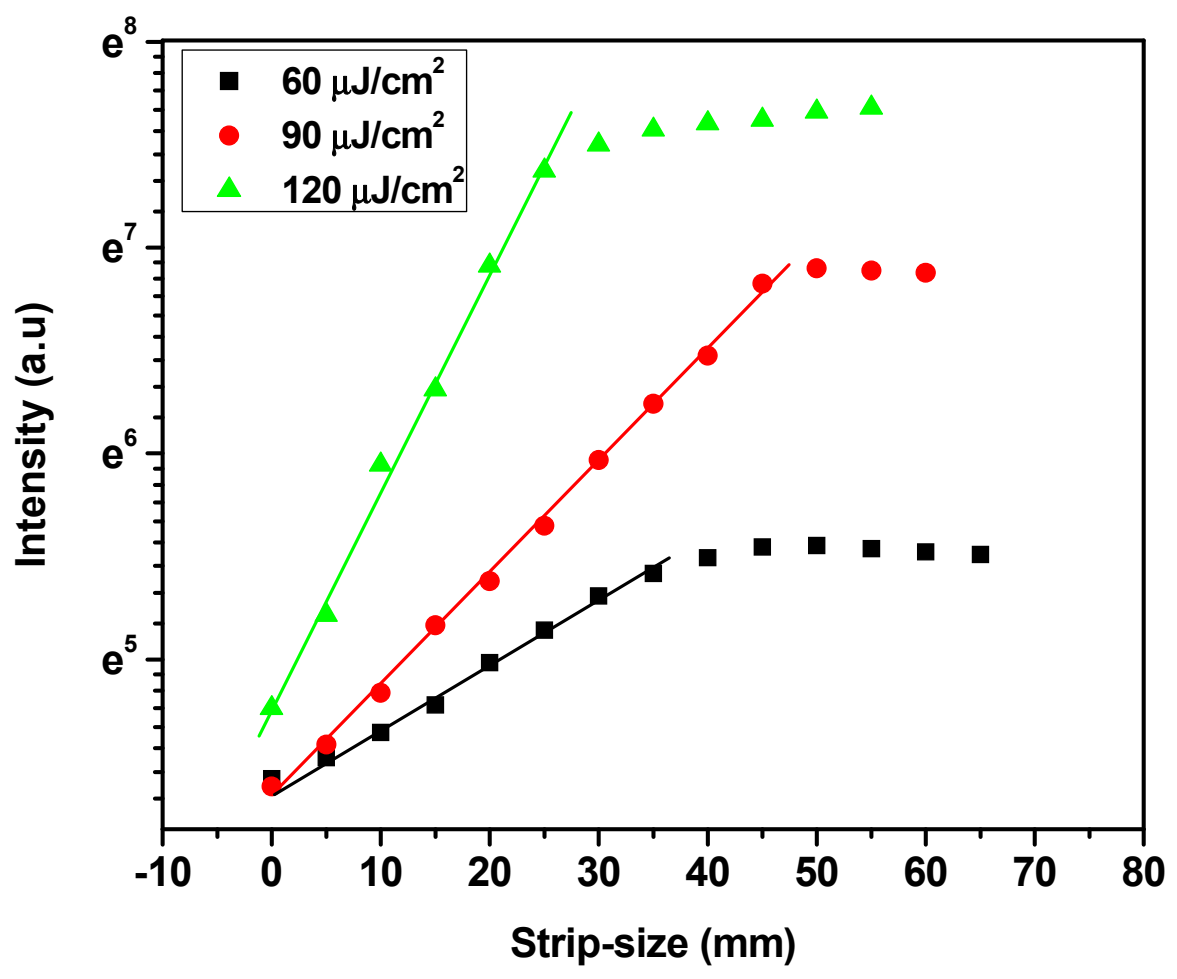

Figure 6. Gain characteristics for P5T waveguide optical amplifiers.

One of the important parameters for photonic materials is the PLQE that justifies the potential of a material for light emitting and laser applications. Figure 7 shows the PLQE measurements of the P5T crystal that exhibits a higher value of $68.24 \%$ than other organic semiconductors such as polyfluorene (PFO) and poly-(2-methoxy-5-(2-ethylhexyloxy)-1,4-phenylenevinylene) (MEH-PPV), which have PLQE values of $51.6 \%$ and $6.8 \%$, respectively [37]. This suggests that P5T is to be effectively used for its potential applications in oligomer-based lasers and OLEFETs.

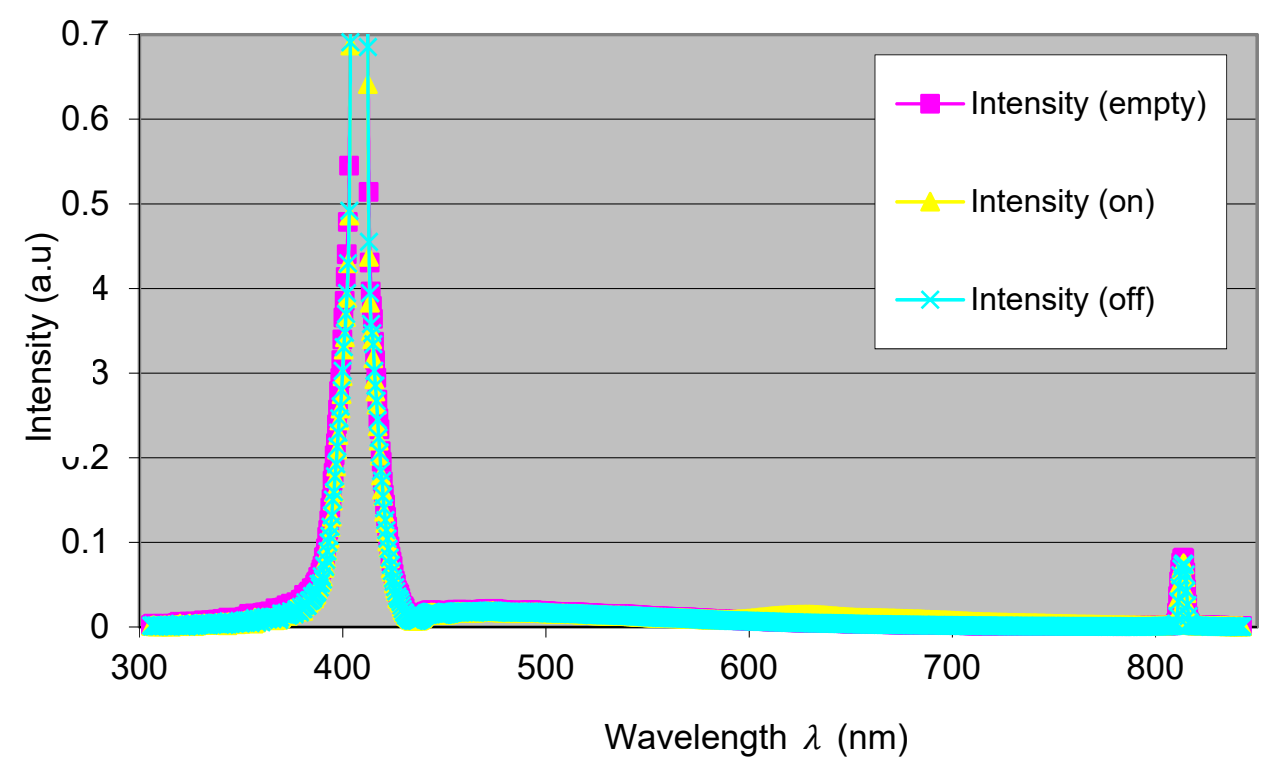

Figure 7. Photoluminescence quantum efficiency (PLQE) of curves of P5T single crystal. 


\section{Conclusions}

In this paper, a thiophene/phenylene co-oligomer single crystal, P5T, has been studied for optical amplification, optical gain, and PLQE measurements. The ASE spectra of P5T crystal were studied within a pump energy range from 35.26 to $163.34 \mu \mathrm{J} / \mathrm{cm}^{2}$ at an excitation wavelength of $445 \mathrm{~nm}$ using a femtosecond-pulsed ND-YAG laser. The threshold energy " $\mathrm{E}_{\text {th }}$ " for the P5T crystal was calculated from the ASE measurements by plotting pump energy as a function of output intensity, and was found to be $52.64 \mu \mathrm{J} / \mathrm{cm}^{2}$. The P5T demonstrated a better and smaller value of $E_{\text {th }}$ for optical amplification and stimulated emission as compared to other organic semiconductors. The optical gain was studied by a VSL technique and the measured value was $72 \mathrm{~cm}^{-1}$ for P5T. A high value of PLQE, i.e., $68.24 \%$, was observed for the crystal. This study endorses the P5T single crystal for its potential use in high performance OLEDs, optical amplifiers, and organic solid state lasers.

Author Contributions: M.Z. and M.T. conceived and designed the experiments; M.Z. and M.T. performed the experiments; M.Z., M.T. and M.R.S. analyzed the data; M.R.S. format the paper. F.M., S.M.S., M.F.M.S., S.H.M.A., F.W. contributed reagents/materials/analysis tools; M.Z. and M.T. wrote the paper.

Funding: This research was funded by the Universiti Kebangsaan, Malaysia, Grant Code DCP-2017-006/2 (UKM) and The APC was funded by DCP-2017-006/2 (UKM).

Acknowledgments: The authors are thankful to the Cavendish Lab, University of Cambridge UK for providing the research facilities, Abdul Wali Khan University Mardan, Pakistan, University of Malaya, Malaysia and Universiti Kebangsaan, Malaysia for funding the research under Grant Code DCP-2017-006/2 (UKM).

Conflicts of Interest: The authors declare no conflict of interest.

\section{References}

1. Huang, C.-C.; Xue, M.-M.; Wu, F.-P.; Yuan, Y.; Liao, L.-S.; Fung, M.-K. Deep-blue and hybrid-white organic light emitting diodes based on a twisting carbazole-benzofuro[2,3-b]pyrazine fluorescent emitter. Molecules 2019, 24, 353. [CrossRef] [PubMed]

2. Liu, N.; Mei, S.; Sun, D.; Shi, W.; Feng, J.; Zhou, Y.; Mei, F.; Xu, J.; Jiang, Y.; Cao, X. Effects of charge transport materials on blue fluorescent organic light-emitting diodes with a host-dopant system. Micromachines 2019, 10, 344. [CrossRef] [PubMed]

3. Bucinskas, A.; Bezvikonnyi, O.; Gudeika, D.; Volyniuk, D.; Grazulevicius, J.V. Methoxycarbazolyl-disubstituted dibenzofuranes as holes- and electrons-transporting hosts for phosphorescent and TADF-based OLEDs. Dye. Pigment. 2020, 172, 107781. [CrossRef]

4. Kim, D.-K.; Choi, J.-H. A study of effects of electrode contacts on performance of organic-based light-emitting field-effect transistors. Opt. Mater. 2018, 76, 359-367. [CrossRef]

5. Jeong, Y.J.; Oh, J.H.; Song, H.J.; An, T.K. A Quinacridone-diphenylquinoxaline-based copolymer for organic field-effect transistors. Polymers 2019, 11, 563. [CrossRef] [PubMed]

6. Gaspar, H.; Figueira, F.; Pereira, L.; Mendes, A.; Viana, J.C.; Bernardo, G. Recent developments in the optimization of the bulk heterojunction morphology of polymer: fullerene solar cells. Materials 2018, 11, 2560. [CrossRef] [PubMed]

7. Carlé, J.E.; Krebs, F.C. Technological status of organic photovoltaics (OPV). Sol. Energy Mater. Sol. Cells 2013, 119, 309-310. [CrossRef]

8. Yan, L.; Qi, M.; Li, A.; Meng, H.; Zhao, X.; Ali, M.U.; Xu, B. Investigating the single crystal OFET and photo-responsive characteristics based on an anthracene linked benzo[b]benzo[4,5]thieno[2,3-d]thiophene semiconductor. Org. Electron. 2019, 72, 1-5. [CrossRef]

9. Sánchez-Vergara, M.E.; Hamui, L.; González Habib, S. New Approaches in flexible organic field-effect transistors (fets) using InClPc. Materials 2019, 12, 1712. [CrossRef]

10. Tahir, M.; Sayyad, M.H.; Wahab, F.; Aziz, F.; Shahid, M.; Munawar, M.A. Perylene diimide: Synthesis, fabrication and temperature dependent electrical characterization of heterojunction with p-silicon. Phys. $B$ Condens. Matter 2013, 426, 6-12. [CrossRef]

11. Tahir, M.; Sayyad, M.H.; Clark, J.; Wahab, F.; Aziz, F.; Shahid, M.; Munawar, M.A.; Chaudry, J.A. Humidity, light and temperature dependent characteristics of $\mathrm{Au} / \mathrm{N}-\mathrm{BuHHPDI} / \mathrm{Au}$ surface type multifunctional sensor. Sens. Actuators B Chem. 2014, 192, 565-571. [CrossRef] 
12. El-Zaidia, E.F.M.; Al-Kotb, M.S.; Yahia, I.S. Physico-chemical properties of acid fuchsin as novel organic semiconductors: Structure, optical and electrical properties. Phys. B Condens. Matter 2019, 571, 71-75. [CrossRef]

13. Jeong, Y.J.; Yun, D.-J.; Nam, S.; Jang, J. Enhanced gate-bias stress stability of organic field-effect transistors by introducing a fluorinated polymer in semiconductor/insulator ternary blends. Appl. Surf. Sci. 2019, 481, 642-648. [CrossRef]

14. Kobayashi, J.; Kawahara, R.; Uchida, S.; Koguchi, S.; Ito, T. Conductive hybrid crystal composed from polyoxomolybdate and deprotonatable ionic-liquid surfactant. Int. J. Mol. Sci. 2016, 17, 994. [CrossRef] [PubMed]

15. Yamao, T.; Ota, S.; Miki, T.; Hotta, S.; Azumi, R. Improved sublimation growth of single crystals of thiophene/phenylene co-oligomers. Thin Solid Films 2008, 516, 2527-2531. [CrossRef]

16. Sasaki, F.; Mori, M.; Haraichi, S.; Ido, Y.; Masumoto, Y.; Hotta, S. Microdisk lasers and field effect transistors of thiophene/phenylene co-oligomers by using high temperature deposition method. Org. Electron. 2010, 11, 1192-1198. [CrossRef]

17. Yamao, T.; Juri, K.; Kamoi, A.; Hotta, S. Field-effect transistors based on organic single crystals grown by an improved vapor phase method. Org. Electron. 2009, 10, 1241-1247. [CrossRef]

18. Yamao, T.; Sakaguchi, T.; Juri, K.; Doi, H.; Kamoi, A.; Suganuma, N.; Hotta, S. Improvements of morphologies and emission characteristics of highly purified organic oligomer semiconductors. Thin Solid Films 2009, 518, 489-492. [CrossRef]

19. Mani, A.; Schoonman, J.; Goossens, A. Photoluminescence study of sexithiophene thin films. J. Phys. Chem. B 2005, 109, 4829-4836. [CrossRef]

20. Bisri, S.Z.; Takenobu, T.; Yomogida, Y.; Shimotani, H.; Yamao, T.; Hotta, S.; Iwasa, Y. High mobility and luminescent efficiency in organic single-crystal light-emitting transistors. Adv. Funct. Mater. 2009, 19, 1728-1735. [CrossRef]

21. Egelhaaf, H.-J.; Gierschner, J.; Oelkrug, D. Characterization of oriented oligo(phenylenevinylene) films and nano-aggregates by UV/Vis-absorption and fluorescence spectroscopy. Synth. Met. 1996, 83, 221-226. [CrossRef]

22. Yamao, T.; Sakurai, Y.; Terasaki, K.; Shimizu, Y.; Jinnai, H.; Hotta, S. Current-injected spectrally-narrowed emissions from an organic transistor. Adv. Mater. 2010, 22, 3708-3712. [CrossRef] [PubMed]

23. Ichikawa, M.; Nakamura, K.; Inoue, M.; Mishima, H.; Haritani, T.; Hibino, R.; Koyama, T.; Taniguchi, Y. Photopumped laser oscillation and charge-injected luminescence from organic semiconductor single crystals of a thiophene/phenylene co-oligomer. Appl. Phys. Lett. 2005, 87, 221113. [CrossRef]

24. Guillén, M.G.; Gámez, F.; Lopes-Costa, T.; Castro-Smirnov, J.R.; Wannemacher, R.; Cabanillas-González, J.; Pedrosa, J.M. Amplified spontaneous emission in action: Sub-ppm optical detection of acid vapors in poly[2-methoxy-5-(2-ethylhexyloxy)-1,4-phenylenevinylene] thin films. Sens. Actuators B Chem. 2018, 255, 1354-1361. [CrossRef]

25. Guzelturk, B.; Kelestemur, Y.; Olutas, M.; Delikanli, S.; Demir, H.V. Amplified spontaneous emission and lasing in colloidal nanoplatelets. ACS Nano 2014, 8, 6599-6605. [CrossRef]

26. She, C.; Fedin, I.; Dolzhnikov, D.S.; Demortière, A.; Schaller, R.D.; Pelton, M.; Talapin, D.V. Low-Threshold stimulated emission using colloidal quantum wells. Nano Lett. 2014, 14, 2772-2777. [CrossRef]

27. Kobayashi, S.; Sasaki, F.; Yanagi, H.; Hotta, S.; Ichikawa, M.; Taniguchi, Y. Spectrally narrowed emissions in 2,5-bis(4-biphenylyl)thiophene crystals pumped by fs laser pulse. J. Lumin. 2005, 112, 325-328. [CrossRef]

28. Anni, M.; Lattante, S. Amplified spontaneous emission optimization in regioregular poly(3-hexylthiophene) (rrp3ht):poly(9,9-dioctylfluorene- co -benzothiadiazole) (f8bt) thin films through control of the morphology. J. Phys. Chem. C 2015, 119, 21620-21625. [CrossRef]

29. Sun, C.; Mróz, M.M.; Castro Smirnov, J.R.; Lüer, L.; Hermida-Merino, D.; Zhao, C.; Takeuchi, M.; Sugiyasu, K.; Cabanillas-González, J. Amplified spontaneous emission in insulated polythiophenes. J. Mater. Chem. C 2018, 6, 6591-6596. [CrossRef]

30. Ichikawa, M.; Yanagi, H.; Shimizu, Y.; Hotta, S.; Suganuma, N.; Koyama, T.; Taniguchi, Y. Organic field-effect transistors made of epitaxially grown crystals of a thiophene/phenylene co-oligomer. Adv. Mater. 2002, 14, 1272-1275. [CrossRef]

31. Yanagi, H.; Morikawa, T.; Hotta, S.; Yase, K. Epitaxial growth of thiophene/p-phenylene co-oligomers for highly polarized light-emitting crystals. Adv. Mater. 2001, 13, 313-317. [CrossRef] 
32. Yamao, T.; Miki, T.; Akagami, H.; Nishimoto, Y.; Ota, S.; Hotta, S. Direct formation of thin single crystals of organic semiconductors onto a substrate. Chem. Mater. 2007, 19, 3748-3753. [CrossRef]

33. Calzado, E.M.; Boj, P.G.; Díaz-García, M.A. Amplified spontaneous emission properties of semiconducting organic materials. Int. J. Mol. Sci. 2010, 11, 2546-2565. [CrossRef] [PubMed]

34. Xia, R.; Heliotis, G.; Stavrinou, P.N.; Bradley, D.D.C. Polyfluorene distributed feedback lasers operating in the green-yellow spectral region. Appl. Phys. Lett. 2005, 87, 031104. [CrossRef]

35. Lampert, Z.E.; Reynolds, C.L.; Papanikolas, J.M.; Aboelfotoh, M.O. Controlling morphology and chain aggregation in semiconducting conjugated polymers: the role of solvent on optical gain in meh-ppv. J. Phys. Chem. B 2012, 116, 12835-12841. [CrossRef]

36. Xia, R.; Heliotis, G.; Hou, Y.; Bradley, D.D.C. Fluorene-based conjugated polymer optical gain media. Org. Electron. 2003, 4, 165-177. [CrossRef]

37. Prakash, A.; Katiyar, M. Correlation between electroluminescence, charge transport and photophysical properties of polymer blends. Synth. Met. 2017, 223, 184-191. [CrossRef]

(C) 2019 by the authors. Licensee MDPI, Basel, Switzerland. This article is an open access article distributed under the terms and conditions of the Creative Commons Attribution (CC BY) license (http://creativecommons.org/licenses/by/4.0/). 